\section{Transcriptional regulation of cardiac genes balance pro- and anti-hypertrophic mechanisms in hypertrophic cardiomyopathy}

\author{
Nina Gennebäck, ${ }^{1}$ Gerhard Wikström, ${ }^{2}$ \\ Urban Hellman, Jane-Lise Samuel, ${ }^{3}$ \\ Anders Waldenström, ${ }^{4}$ Stellan Mörner ${ }^{4}$ \\ ${ }^{1}$ Department of Public Health and Clinical \\ Medicine/Medicine, Umea University, \\ Umea, Sweden; ' $D$ epartment of Medical \\ Sciences, Cardiology, Uppsala University, \\ Uppsala, Sweden; 'INSERM U 942, 41, \\ Bd de la Chapelle, Paris, France, Hôpital \\ Lariboisière Paris France; ${ }^{4}$ Department \\ of Cardiology, Heart Centre, Umeå \\ University Hospital and Department \\ of Public Health and Clinical Medicine, \\ Umeå University, Umeå, Sweden
}

\section{Abstract}

Hypertrophic cardiomyopathy (HCM) is characterized by unexplained left ventricular hypertrophy. HCM is often hereditary, but our knowledge of the mechanisms leading from mutation to phenotype is incomplete. The transcriptional expression patterns in the myocardium of HCM patients may contribute to understanding the mechanisms that drive and stabilize the hypertrophy.

Cardiac myectomies/biopsies from 8 patients with hypertrophic obstructive cardiomyopathy (HOCM) and 5 controls were studied with whole genome Illumina microarray gene expression (detecting $18189 \mathrm{mRNA}$ ).

When comparing HOCM myocardium to controls, there was significant transcriptional down-regulation of the $M Y H 6, E G R 1, A P O B$ and $F O S$ genes, and significant transcriptional up-regulation of the ACE2, JAK2, NPPA (ANP), $A P O A 1$ and HDAC5 genes.

The transcriptional regulation revealed both pro- and anti-hypertrophic mechanisms. The pro-hypertrophic response was explained by the transcriptional down-regulation of $M Y H 6$, indicating that the switch to the fetal gene program is maintained, and the transcriptional up-regulation of JAK2 in the JAK-STAT pathway. The anti-hypertrophic response was seen as a transcriptional down-regulation of the immediate early genes (IEGs), FOS and EGR1, and a transcriptional up-regulation of $A C E 2$ and HDAC5. This can be interpreted as a transcriptional endogenous protection system in the heart of the HOCM patients, neither growing nor suppressing the already hypertrophic myocardium.

\section{Introduction}

Hypertrophic cardiomyopathy (HCM) is characterized by cardiac hypertrophy of the septum and/or left ventricular walls in the absence of other pro-hypertrophic diseases. ${ }^{1}$ The disease is often monogenetic, caused by mutations in sarcomeric genes in approximately $56 \%$ of cases. ${ }^{2}$ At the cellular level, there is an increase in cardiomyocyte size, myocyte disarray and fibrosi..$^{3-4} \mathrm{HCM}$ is associated with an increased risk of mortality and morbidity and is a common cause of sudden cardiac death in young adults. ${ }^{5}$ In a subset of patients with hypertrophic obstructive cardiomyopathy (HOCM), surgical myectomy may be indicated to alleviate symptoms.

To date, numerous signalling pathways and transcription factors have been related to cardiac hypertrophy, including the janus kinase signal transducer and activator of transcription (JAK-STAT) pathway, ${ }^{6}$ the fetal gene program ${ }^{7}$ and transcription influenced by the immediate early genes. ${ }^{8}$ Analysis of the transcriptome can be made by using microarray gene expression techniques which give a picture of the gene activity at a given time point. In HCM, such studies have been carried out in experimental animal models as well as in human hearts. In 2001, Lim et al. performed a DNA subtraction hybridization analysis of tissue from septal myectomies in a single human heart with HCM and from a normal heart, followed by confirmation of the results in 6 more HCM hearts. Upregulation of markers of cardiac hypertrophy, such as alpha skeletal actin, isoforms of myosin light chains and brain natriururetic peptide was found.$^{9}$ In 2002, Hwang et al. investigated the gene expression in end-stage heart failure due to HCM (2 patients) or dilated cardiomyopathy (DCM) (3 patients) by using an in-house spotted cDNA microarray with 10,272 unique clones from various cardiovascular cDNA libraries in their laboratory. A total of 192 genes were highly expressed in both HCM and DCM (e.g. ANP, decorin, elongation factor 2 and heat shock protein 90 ) and 51 genes were down-regulated in both conditions (e.g. elastin and sarco-/endoplasmic reticulum Ca ATPase, SERCA). ${ }^{10}$ Rajan et al. studied ventricular tissue from two different transgenic mouse models carrying mutations in the alpha-tropomyosin gene (TPM1). Out of 22,600 genes studied, 754 were differentially regulated in the transgenic mice compared to controls. Genes with the highest expression belonged to secreted/extracellular matrix category and genes with the most significant decrease were associated with metabolic enzymes. ${ }^{11}$ Thus, a number of differentially expressed genes have been identified in HCM.

In HCM, cardiac hypertrophy often progresses for a number of years and then subsides, as shown in a study by Maron et al. where left
Correspondence: Nina Gennebäck, Department of Public Health and Clinical Medicine/Medicine Umeå University, S-901 87 Umeå, Sweden. Tel. +46.0.90.785 4483 - Fax: +46.0 .90 .130760 . E-mail: nina.genneback@medicin.umu.se

Key words: hypertrophic cardiomyopathy, HCM, gene expression, microarray.

Acknowledgements: this research received financial support from the Swedish Heart Lung Foundation and the Heart Foundation of Northern Sweden.

Contributions: NG participated in the design of the study, carried out the micro array study, the statistical and bioinformatical analysis and drafted the manuscript. GW and J-LS provided patient material, conceived of the study. UH, AW and SM conceived of the study, participated in its design and coordination and helped to draft the manuscript. All authors read and approved the final manuscript.

Conflict of interests: the authors have no conflict of interests.

Received for publication: 10 November 2011

Revision received: 25 April 2012.

Accepted for publication: 27 April 2012

This work is licensed under a Creative Commons Attribution NonCommercial 3.0 License (CC BYNC 3.0).

(C) Copyright N. Gennebäck et al., 2012

Licensee PAGEPress, Italy

Cardiogenetics 2012; 2:e5

doi:10.4081/cardiogenetics.2012.e5

ventricular wall thickness increased by 6 to 23 mm during a mean follow up of four years in children with HCM. ${ }^{12}$ It is important to identify how different pathways interact to cause, control and maintain the hypertrophy so that the progression of the heterogeneous disease can be better understood and a target for future therapy might be developed depending on the stage of hypertrophy. Today, the whole transcriptome can be assessed at once by the use of whole genome microarray analysis, which has proven to be useful in portraying molecular events in a specific tissue sample. It is not known what starts the hypertrophic process in HCM, nor is it known what stops it in order to prevent growth in absurdum. By combining results from animal experiments and human material from patients from different stages of the disease, microarray data might shed light on what starts the process. The aim of this study was to examine the transcriptional expression in the myocardium of patients with symptomatic HOCM in an attempt to explain the mechanisms that drive and maintain the hypertrophy. 


\section{Materials and Methods}

\section{Study population}

This study includes myectomies from 8 unrelated patients with non-end stage HOCM. The myectomies were collected between November 2005 and April 2007 on obtaining written consent. The HOCM patients were diagnosed using criteria proposed by McKenna et al..$^{13}$ Three of the patients were also diagnosed with mild hypertension, although this was not sufficient to cause this severe phenotype. The electrocardiographic left ventricular hypertrophy (LVH) was interpreted using the Romhilt-Estes score. ${ }^{14}$ As controls, myectomies/biopsies were collected from 5 respirator patients, without cardiac disease who were deceased following accidents. Consent was given to the hospital administration of Hôpital Lariboisière in Paris according to French legislation. The study was approved by the ethical committees of Umeå, Uppsala and Paris.

\section{Sample preparation}

The myectomies/biopsies from the patients were excised and immediately placed in RNAlater (Qiagen, Hilden, Germany). Total RNA was isolated using the RNeasy Fibrous Tissue Kit (Qiagen, Stanford, CA, USA). The RNA concentration was measured with a NanoDrop ND-1000 Spectrophotometer (NanoDrop Technologies, Inc, Wilmington, USA) and the integrity of the RNA was analyzed with a 2100 Bioanalyzer (Agilent Technologies Inc, Palo Alto, CA, USA).

\section{Microarray gene expression}

Using the Illumina Totalprep RNA Amplification Kit (Ambion, Austin, TX, USA), aliquots of total RNA were converted to biotinylated double-stranded cRNA. The biotin labeled cRNA samples were hybridized on a Sentrix HumanRef-8 Expression Beadchip (Illumina, San Diego, CA, USA), including 18,189 gene transcripts, and incubated with streptavidinCy3. The microarray analysis was scanned with the Illumina Beadstation GX (Illumina).

\section{Data analysis}

To find differentially expressed genes in the microarray data, Illumina GenomeStudio software (version 3.3.8) was used. The data were normalized and significant differential expression was calculated using Beadstudios cubic spline algorithm. The gene expression fold change for the patient group was calculated as the average signal value relative to the average signal value for the control patient group. A significant up-regulation was defined as a fold change of 1.5 or over and a significant downregulation was defined as fold change of -1.5 or under. $\mathrm{P}<0.05$ was considered statistically sig- nificant. To avoid selecting genes with high fold change due to low signal intensity a minimum signal intensity value was used; the signal intensity was set at over 50 . The statistical filtering can be found in Table 1 .

MetaCore $^{\mathrm{TM}}$ (GeneGo Inc., USA) was used to find common transcriptional relations between transcription factors and genes in HCM to generate a direct interaction network.

\section{Quantitative real time polymerase chain reaction}

To validate the microarray expression data, we performed relative quantification of mRNA expression of four genes, differentially expressed in the microarray analysis using an Applied Biosystems Prism 7900HT Sequence Detection System (Foster City, CA, USA). Omniscript RT Kit (Qiagen) was used to convert an aliquot of the remaining total RNA to cDNA. Human cDNA-specific TaqMan Gene Expression Assays for the genes: angiotensin converting enzyme 2 (ACE2) (Assay ID Hs01085333_m1), apolipoprotein A-I (APOA1) (Assay ID Hs00163641_m1), early growth response 1 (EGR1) (Assay ID Hs00152928_ml) and myosin heavy chain 6 cardiac muscle alpha (MYH6) (Assay ID Hs00411908_ml) from Applied Biosystems were used in the study. The human cyclophilin A (PPIA) gene was chosen as an endogenous control (part n. 432631E; Applied Biosystems Inc, Foster City, CA, USA) after confirmation as the most stable in myectomies when comparing with glyceraldehyde-3phosphate dehydrogenase (GADPH) and large ribosomal protein (RPLPO). ${ }^{15}$ All samples were run in triplicates and amplification was analyzed using Applied Biosystems Prism Sequence Detection software (version 2.3). Relative quantification was calculated according to the comparative CT method (Applied Biosystems Inc) using a statistical confidence of $95 \%$. The amount of target gene mRNA, normalized to an endogenous control and relative

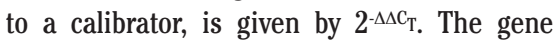
expression fold change of the patients is the average $2^{-\Delta \Delta \mathrm{C}_{\mathrm{T}}}$ value relative to the average 2 $\Delta \Delta \mathrm{C}_{\mathrm{T}}$ value for the control patients.

\section{Statistics}

All analyses of the real time polymerase chain reaction (PCR) data were performed with Predictive analytics software (PASW) (version 18.0, SPSS Inc., Chicago, Ill, USA). Differences between two groups were compared using Mann-Whitney $\mathrm{U}$ test. $\mathrm{P}<0.05$ was considered statistically significant.

\section{Multivariate data analysis}

SIMCA-P+ 12.0.1 software (Umetrics, Umeå, Sweden) was used to perform principal component analysis (PCA) to find ${ }^{16-17}$ clusters and outliers in the sample population. A PCA model was calculated on the X-matrix (i.e. the microarray data with the signal intensity $>50$ ) to provide an overview of the data. In the data matrix $\mathrm{X}$ [ $\mathrm{NxK}], \mathrm{N}$ defines the number of rows or samples and $K$ the number of variables or gene transcripts. A small number of latent variables (principal components) are calculated; these variables reflect the largest systematic variations by describing the largest variation in the X-matrix. As a result, the influence of noise is reduced and the dimensionality of the data is greatly reduced, which simplifies interpretation. Scores are the coordinates of the samples in the reduced space (the principal component space) whereas loadings are the relations between the original variables and the principal components. In the score plots, trends and outliers can be found and clusters of samples can be revealed.

\section{Results}

\section{Clinical analysis of cohort}

The demographics and patient history of the HOCM cohort ( $\mathrm{n}=8 ; 3$ men and 5 females) are summarized in Tables 2 and 3. The mean age at diagnosis was $60.5 \pm 17.0$ years with a mean maximum wall thickness of $18.6 \pm 2.5 \mathrm{~mm}$ and a mean left ventricular outflow tract-obstruction gradient of $79 \pm 46 \mathrm{mmHg}$. The mean NT pro BNP was $5253 \pm 8906 \mathrm{ng} / \mathrm{L}$. Information on cause of death, age and sex of the control patient group can be found in Table 4. All patients had a preserved systolic left ventricular function with normal fractional shortening.

Table 1. Number of differentially expressed genes after different filtering steps.

\begin{tabular}{|c|c|c|}
\hline Filtrating step & \multicolumn{2}{|c|}{$\begin{array}{l}\text { mRNA expression } \\
\text { Number of genes }\end{array}$} \\
\hline $\begin{array}{l}\text { Differential } \\
\text { P-value }<0.05\end{array}$ & $343 \uparrow$ & $254 \downarrow$ \\
\hline & \multicolumn{2}{|c|}{$\downarrow$} \\
\hline $\begin{array}{l}\text { Detection } \\
\text { P-value }<0.05\end{array}$ & $330 \uparrow$ & $233 \downarrow$ \\
\hline $\begin{array}{l}\text { Avg. sign. } \\
\text { P-value }>50\end{array}$ & $330 \uparrow$ & $233 \downarrow$ \\
\hline & \multicolumn{2}{|c|}{$\downarrow$} \\
\hline $\begin{array}{l}\text { Fold change } \\
(\mathrm{mRNA}) \pm 1.5\end{array}$ & $283 \uparrow$ & $210 \downarrow$ \\
\hline
\end{tabular}

Statistical significance was set to $\mathrm{P}<0.05$. A significant up- and downregulation was defined as a fold change \pm 1.5 . A minimum signal intensity value of 50 was utilized. 
Table 2. Patient characteristics.

\begin{tabular}{|c|c|c|c|c|c|c|c|c|c|c|c|}
\hline Sex & ID & Age (yrs) & $\begin{array}{l}\text { Blood } \\
\text { pressure } \\
\text { (mmHg) }\end{array}$ & $\mathrm{ECG}^{20}$ & $\begin{array}{c}\text { NTpro } \\
\text { BNP }\end{array}$ & $\begin{array}{c}\mathrm{LA} \\
(\mathrm{mm})\end{array}$ & $\begin{array}{l}\text { IVSD } \\
(\mathrm{mm})\end{array}$ & $\begin{array}{l}\text { LVPWD } \\
(\mathrm{mm})\end{array}$ & $\begin{array}{l}\text { Max. wall } \\
\text { thickness } \\
(\mathrm{mm})\end{array}$ & $\begin{array}{l}\text { LVEDD } \\
(\mathrm{mm})\end{array}$ & $\begin{array}{l}\text { LVOT- } \\
\text { obstruction } \\
\text { (mmHg) }\end{array}$ \\
\hline Woman & $\mathrm{Hl}$ & 75 & $150 / 75$ & LVH & $1181^{*}$ & 47 & 16 & 10 & 16 & 44 & 60 \\
\hline Man & $\mathrm{H} 2$ & 51 & $145 / 80$ & LVH & $295^{*}$ & 49 & 18 & 15 & 19 & 51 & 36 \\
\hline Woman & $\mathrm{H} 3$ & 79 & $150 / 75$ & LVH & $1412^{*}$ & 53 & 17 & 17 & 17 & 45 & 159 \\
\hline Man & $\mathrm{H} 4$ & 50 & $130 / 80$ & LVH, QT-prolongation & 25139 & 95 & 18 & 15 & 20 & 50 & 16 \\
\hline Woman & $\mathrm{H} 5$ & 70 & $100 / 60$ & LVH & - & 46 & 16 & 15 & 19 & 39 & 117 \\
\hline Woman & $\mathrm{H} 6$ & 76 & $130 / 70$ & LBBB & - & 39 & 13 & 11 & 15 & 42 & 64 \\
\hline Man & $\mathrm{H} 7$ & 31 & $130 / 70$ & LVH & 1633 & 38 & 22 & 12 & 22 & 50 & 91 \\
\hline Woman & $\mathrm{H} 8$ & 52 & $130 / 80$ & LVH, inferior Q-wave & 1862 & 44 & 12 & 10 & 21 & 44 & 89 \\
\hline
\end{tabular}

ECG, Electrocardiography; LA, left atrium; IVSD, interventricular septum dimension; LVPWD, left ventricular posterior wall dimension; LVEDD, left ventricular end diastolic diameter; LVOT, left ventricular outflow tract; LVH, left ventricular hypertrophy; LBBB, left bundle branch block. "post myectomy.

\section{Sample preparation}

The integrity analysis of the RNA from both patients and controls showed no breakage or digestion.

\section{Microarray gene expression}

The microarray data was filtrated as described in the Methods section (Table 1). There were 493 gene transcripts (283 were upregulated and 210 were down-regulated) that were found to be significantly differentially regulated in patients compared to controls. The MetaCore ${ }^{\mathrm{TM}}$ (GeneGo Inc., USA) bioinformatics software was used to find genes with correlation to heart disease, hypertrophy and transcriptional changes among the 493 gene transcripts. Relevant regulated gene transcripts were the up-regulation of ACE2, JAK2, and the down-regulation of EGR1, FOS and MYH6 (Table 5). MetaCore ${ }^{\mathrm{TM}}$ was also used to generate a direct interaction network. The direct interaction network analysis resulted in a network with direct connection between 101 of the $493(\sim 20.5 \%)$ significant transcripts, the network was completed by adding CCAAT/enhancer-binding protein alpha (C/EBPalpha), EGR1 and ATP-binding cassette, sub-family A (ABCA1) (Figure 1). All data are MIAME compliant and available through NCBIs Gene Expression Omnibus (GEO) (GEO Series accession n. GSE32453).

\section{Quantitative real time polymerase chain reaction}

The levels of ACE2, APOA1 EGR1 and MYH6 mRNA were measured using real time PCR (Table 6). Both the fold change and the P values showed higher significance in 3 cases (MYH6, APOA1 and ACE2) than with the Illumina microarray beadchip. In one case (EGR1), the fold change showed a similar down-regulation while the $P$ value was not significant. Further real time polymerase chain reaction (RT-PCR) analysis was not possible due to the limited size of biopsies/myectomies.

Table 3. Patient medications and other diseases.

\begin{tabular}{lll} 
ID & Other diseases & Medication \\
H1 & $\begin{array}{l}\text { Diabetes mellitus, } \\
\text { arterial hypertension }\end{array}$ & $\begin{array}{l}\text { Candesartan/ hydrochlorothiazide, metoprolol, simvastatin, } \\
\text { metformin, insulin }\end{array}$ \\
H2 & $\begin{array}{l}\text { Diabetes mellitus, } \\
\text { hypercholesterolemia, } \\
\text { arterial hypertension }\end{array}$ & Metoprolol, simvastatin, ramipril, insulin \\
\hline H3 & Atrial fibrillation & Acetylsalicylic acid, metoprolol \\
H4 & Arterial hypertension & Acetylsalicylic acid, metoprolol, losartan \\
H5 & $\begin{array}{l}\text { Hypercholesterolemia, } \\
\text { angina pectoris }\end{array}$ & Metoprolol, acetylsalicylic acid, atorvastatin \\
H6 & $\begin{array}{l}\text { Diabetes mellitus, } \\
\text { previous myocardial infarction }\end{array}$ & Metformin, quinapril, acetylsalicylic acid, simvastatin \\
\hline H7 & - & Metoprolol \\
H8 & - & Bisoprolol, losartan
\end{tabular}

\section{Multivariate data analysis}

A PCA analysis was performed to clarify whether patient history (e.g. medication, other diseases, age or sex) affects the gene expression result in such a way that clusters of individuals can be traced back to factors other than the disease (Figure 2). The PCA analysis revealed a dispersed control group and three clusters in the HOCM patient group (Figure 2).

\section{Discussion}

We studied the transcriptional expression in the myocardium of patients with symptomatic HOCM. The results are compared with known gene expression responses in cardiac hypertrophy to generate a hypothesis explaining the mechanisms that drive and maintain the pathological growth, leading to a balanced chronic hypertrophy.

Due to limited access to samples (especially control tissue), myocardial gene expression data in human HCM is scarce, performed on very few patients in different stages of the disease, with somewhat diverging methodology and results. However, there have been some common findings such as increased expression of natriuretic peptide genes. Our whole genome expression study on 8 non-end stage HOCM patients provides additional information on this subject. Although lacking the support of longitudinal data, we present a hypothesis that when expressions of pro- and anti hypertrophic genes are in equilibrium, a steady state is achieved with no further development of hypertrophy.

In the characterization (network and biomarker analysis) of the 493 differentially expressed transcripts, 101 (20.5\%) were connected with direct interactions indicating a common transcriptional regulation (Figure 1). The result also revealed significant regulation of two important biomarkers for cardiac hypertrophy: MYH6 and ANP. This characterization validates microarray as an established method and indicates that the findings are associated to the hypertrophic process in the myocardium of the HOCM patients.

To evaluate the influence of patient history (e.g. age, sex, medication, other diseases) on the observed gene expression patterns in HOCM, a multivariate data analysis was per- 
formed. The analysis showed that patient history can not be associated to the differentially expressed gene transcripts (Figure 2A and B, Tables 2 and 3 ), indicating that the differences seen are related to the disease. The fact that fractional shortening was normal in all patients suggests that systolic function was preserved and that the differential gene expression seen is due to the hypertrophy itself and not to systolic dysfunction.

The patients in the control group have a somewhat lower age range than the patients in the HOCM group (Tables 2 and 4); nevertheless, the PCA analysis revealed no clustering according to age, indicating that age is not a factor for separating the groups. The same can be concluded about the 3 HOCM patients with hypertension (Table 3), no clustering according to blood pressure could be found.

Data regarding the underlying pathophysiological mechanisms in HCM are diverging, but one of the current hypotheses suggests that the dysfunction of the sarcomere may lead to a rightward shift on the Frank Starling curve and thus increased wall stress. The adaptive response of the cardiomyocyte would be to initiate pro-hypertrophic signals, where two important, initial responses in a sequence may be the secretion of angiotensin II (AngII) ${ }^{18}$ and endothelin 1-3 (EDN1, EDN2 and EDN3) ${ }^{18}$ After this initial response an increased expression of genes would be a second response. In this study, the $A C E$ gene and the endothelin gene transcripts (EDN1, EDN2 and EDN3) were not differentially expressed compared to controls, which indicates that the initial hypertrophic response is no longer present. Another early response at the transcriptional level is the activation of the immediate early genes (IEGs), which are known as early regulators of cell growth and differentiation activated in response to stimuli mediated by AngII and/or other mechanical factors. ${ }^{19}$ IEGs are the first response to stimuli, activating the genome, before the onset of protein synthesis. Examples of known IEGs are, EGR1, FOS and
$J U N$. In our study the IEGs were either downregulated (FOS and EGRI) or not differentially regulated at all (JUN and JUNB), indicating a reduced need for IEGs in this stage of the hypertrophic process. An additional transcriptional regulator is histone deacetylase 5 (HDAC5), which is known to repress the expression of pro-hypertrophic genes, such as myocyte enhancer factor-2 (MEF2). MEF2 was

Table 4. Control patient characteristics.

\begin{tabular}{lccl} 
Sex & ID & $\begin{array}{c}\text { Age } \\
\text { (years) }\end{array}$ & $\begin{array}{c}\text { Cause } \\
\text { of death }\end{array}$ \\
Man & C1 & 46 & CO intoxication \\
Man & C2 & 37 & Head trauma \\
\hline Woman & C3 & 31 & Head trauma \\
Man & C4 & 29 & Traffic accident \\
\hline Man & C5 & 58 & Head trauma \\
\hline
\end{tabular}

Table 5. Gene characteristics and results of gene expression.

\begin{tabular}{|c|c|c|c|c|}
\hline & & & & GEX \\
\hline Design & & Description & P-value & Fold change \\
\hline$A C E$ & $\downarrow$ & Angiotensin I converting enzyme (peptidyl-dipeptidase A) 1 & 0.968 & -1.09 \\
\hline ACE2 & $\uparrow$ & Angiotensin I converting enzyme (peptidyl-dipeptidase A) 2 & 0.0016 & 6.50 \\
\hline ACTA2 & $\uparrow$ & Actin, alpha, cardiac muscle 1 & 0.739 & 1.07 \\
\hline ACTC1 & $\downarrow$ & Actin, alpha 2, smooth muscle, aorta & 0.907 & -1.02 \\
\hline EDN1 & $\downarrow$ & Endothelin 1 & 0.703 & -1.18 \\
\hline EDN2 & $\downarrow$ & Endothelin 2 & 0.648 & -1.30 \\
\hline EDN3 & $\downarrow$ & Endothelin 3 & 0.708 & -51.19 \\
\hline EGR1 & $\downarrow$ & Early growth response 1 & 0.057 & -2.27 \\
\hline FOS & $\downarrow$ & $\mathrm{v}$-fos FBJ murine osteosarcoma viral oncogene homolog & 0.0007 & -9.57 \\
\hline HDAC5 & $\uparrow$ & Histone deacetylase 5 & 0.008 & 2.52 \\
\hline$J A K 2$ & $\uparrow$ & Janus kinase 2 (a protein tyrosine kinase) & 0.00001 & 2.85 \\
\hline JUN & $\downarrow$ & Jun oncogene & 0.216 & -1.34 \\
\hline$J U N B$ & $\downarrow$ & Jun B proto-oncogene & 0.892 & -1.26 \\
\hline MYH6 & $\downarrow$ & Myosin, heavy chain 6 , cardiac muscle, alpha & 0.000005 & -3.51 \\
\hline MYH7 & $\downarrow$ & Myosin, heavy chain 7, cardiac muscle, beta & 0.86 & -1.03 \\
\hline$N P P A$ & $\uparrow$ & Natriuretic peptide precursor A & 0.029 & 6.28 \\
\hline$N P P B$ & $\uparrow$ & Natriuretic peptide precursor B & 0.340 & 2.30 \\
\hline
\end{tabular}

$\uparrow$ and $\downarrow$ indicates change in gene expression in patients compared to controls. GEX, gene expression.

Table 6. Gene characteristics and results of real time polymerase chain reaction analysis in patients $v s$. controls.

\begin{tabular}{|c|c|c|c|c|c|}
\hline Designation & Description & Accession number & $\begin{array}{l}\text { Applied biosystems } \\
\text { assay number }\end{array}$ & P-value & Fold change \\
\hline ACE2 & Angiotensin I converting enzyme 2 & NM_021804.2 & Hs01085333_m1 & 0.005 & 5.42 \\
\hline APOA1 & Apolipoprotein A-I & NM_000039.1 & Hs00163641_ml & 0.048 & 2.84 \\
\hline EGR1 & Early growth response 1 & NM_001964.2 & Hs00152928_ml & 0.202 & -2.18 \\
\hline MYH6 & Myosin, heavy chain 6 , cardiac muscle, alpha & NM_002471.2 & Hs00411908_ml & 0.018 & -4.07 \\
\hline
\end{tabular}


not differentially regulated). ${ }^{20}$ In the study, HDAC5 was up-regulated indicating activation of an anti-hypertrophic program. These results show that the early and instant hypertrophic response is not present in the HOCM patients, suggesting that the acute response is attenuated and an anti-hypertrophic pattern of the transcriptional regulation is detected. This is in agreement with the fact that the disease is at a significant stage and required surgical intervention (myectomy).

At birth, there is a switch from the fetal gene program which involves the regulation of a number of different genes, ${ }^{7,21-23}$ including MYH6, skeletal -actin (ACTA1) and natriuretic peptide precursor A (NPPA). Part of the adaptation to increased workload is the development of hypertrophy and reinduction of the fetal gene program, including a relative increase of MYH7 compared to $\mathrm{MYH6}$. We found $M Y H 6$ to be significantly down-regulated in the HOCM patients (with preserved systolic function). Similar regulations have also been shown in studies of cardiac failure. ${ }^{22-23}$ MYH6 is regulated, amongst other, by the transcription factor $E G R 1^{24}$ which was transcriptionally down-regulated in the present study, which could be a cause for the low expression of $M Y H 6$. Another component of the fetal gene program is the increased expression of NPPA, commonly regarded as driven by the RAAS activation. In our data, NPPA was significantly upregulated and natriuretic peptide precursor $B$ (NPPB) was non-significantly up-regulated to the same level with corresponding increases in serum levels of NT pro BNP (Table 2). This upregulation of NPPA is seen despite a possible counter acting RAAS blockade by $A C E$ inhibitors or angiotensin II type 1 receptor blockade (ARB) in 5 of the HOCM patients. Another regulation of the fetal gene program, that is normally seen is that from cardiac (ACTC1) to skeletal (ACTA1) $\alpha$-actin. ${ }^{25}$ In our patients, this switch was not detected. Together, these results suggest that the fetal gene program is still partially activated even in the chronic state HOCM.

Several signalling pathways have been identified to contribute to the increased gene expression and protein synthesis in cardiac hypertrophy. Examples of such pathways are the JAK-STAT pathway, the $\mathrm{Ca}^{+}{ }^{+}$calmodulin
(CaM)-dependent calcineurin pathway and the nuclear factor kappa-light-chain-enhancer of activated B cells (NF- $\kappa \mathrm{B})$ pathway. ${ }^{21}$ In this study, the only differentially regulated signalling pathway detected was the JAK-STAT pathway (where JAK2 was up-regulated). $J A K 2$, previously shown to have a parallel activation to the onset of cardiac hypertrophy, has an important role in mediating signals from the sarcolemma cytokine gp130 receptor ${ }^{26}$ and angiotensin receptor $\mathrm{AT}_{1}{ }^{27}$ to the nucleus for transcriptional regulation. The results suggest that maintenance of hypertrophy seen in our patients might well be preserved mainly via the JAK-STAT pathway.

The RAAS is known as a regulator of cardiac growth. Ang II is a pro-hypertrophic octapeptide formed by conversion of the decapeptide angiotensin I (Ang I) by angiotensin-converting enzyme ( $A C E)$. Angiotensin 1-7 (Ang 1-7) is a peptide that exerts actions opposite to those of Ang II and is formed by conversion of Ang I by $A C E 2 .{ }^{28} \mathrm{An}$ increased $A C E$-activity may contribute to the harmful effects of RAAS in cardiac diseases. The purpose of an activated local RAAS might be to assist in the develop-

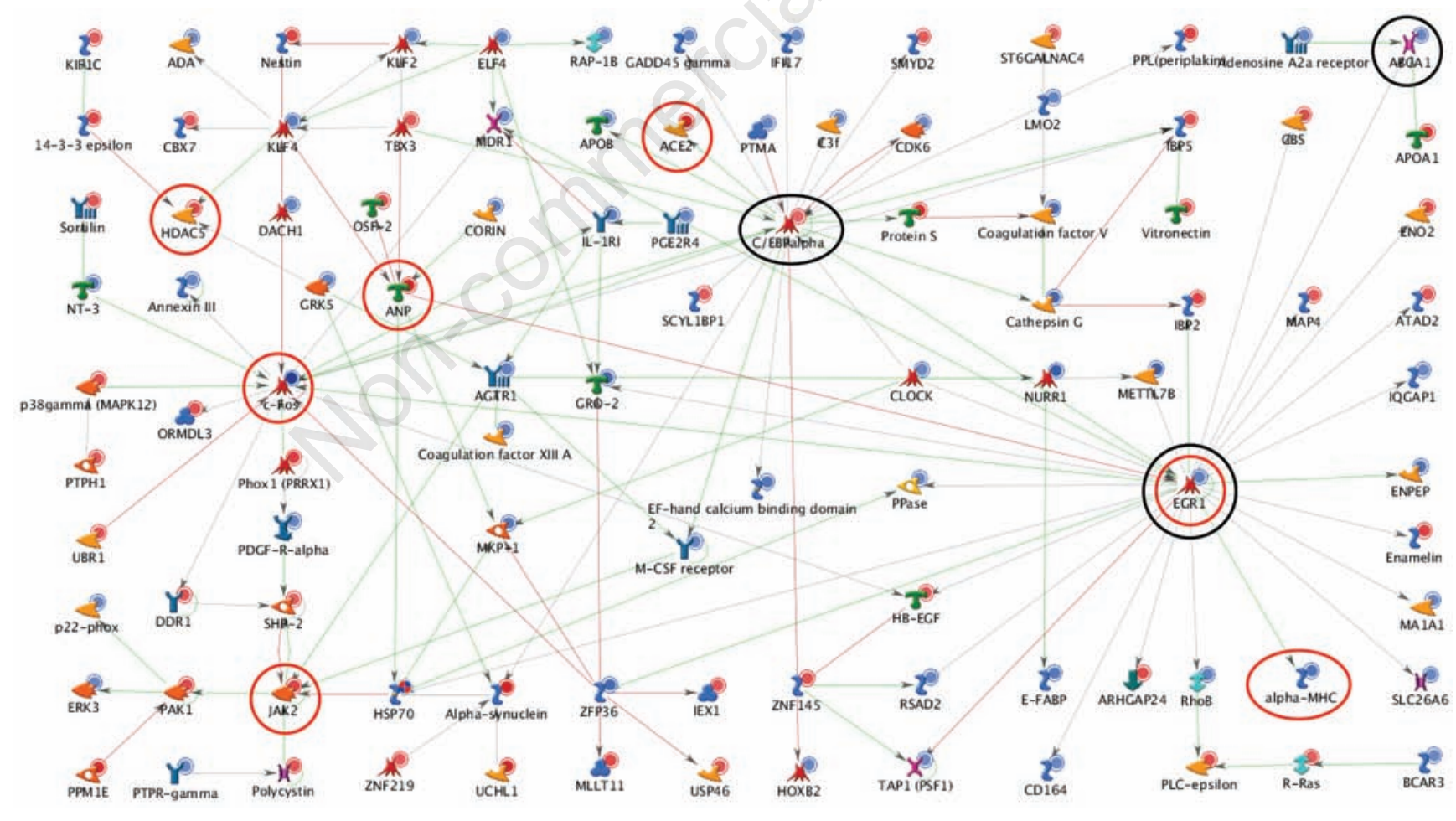

Figure 1. MetaCore ${ }^{\mathrm{TM}}$ direct interaction network analysis. 101 of the $493(-20.5 \%)$ differentially expressed transcripts could be connected by direct interaction in the network analysis. Encircled with red are important genes and transcription factors, further discussed in the discussion. Encircled with black is not present in the 493 transcripts. Green arrows indicate activation and red arrows inhibition. The symbols are as follows: $\theta$ transcriptional up-regulation; $\theta$ transcriptional down-regulation; $\ll$ enzyme; $*$ kinase; $<$ protease; $\varangle$ phosphatase; $\$$ protein; 2 generic binding protein; 4 transcription factor; $\$$ GTPase; $\leftrightarrow$ G-protein; ${ }^{\star}$ G-protein adaptor; $Y$ receptor; $T$ receptor ligand; $x$ channel; $X$ transporter. 


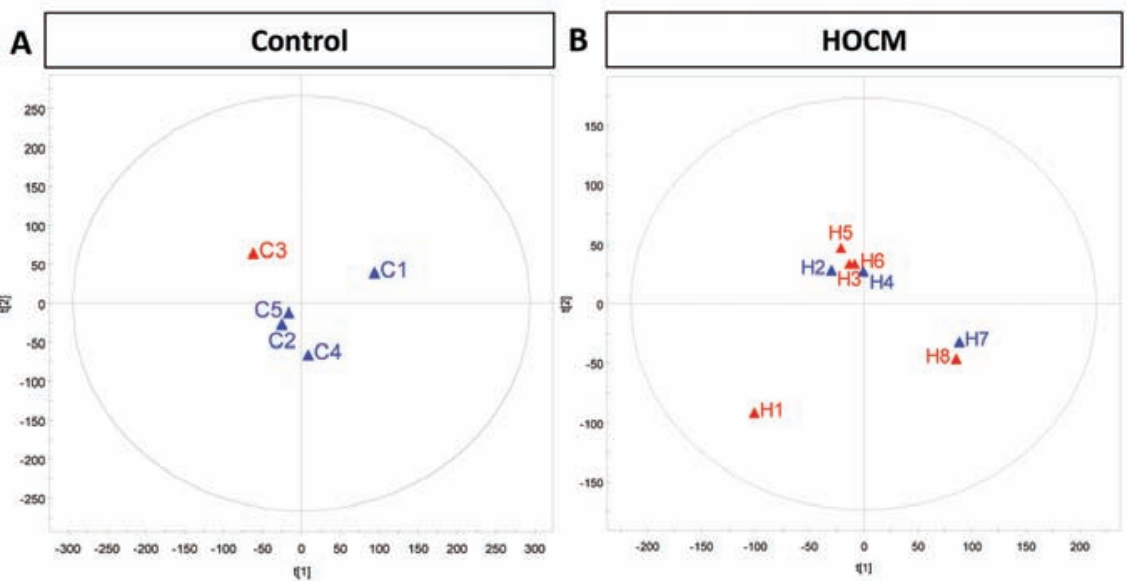

Figure 2. The PCA analysis revealed no groupings in the control group (A, control patient history can be found in Table 4) and three clusters in the hypertrophic obstructive cardiomyopathy (HOCM) patient group (B, HOCM patient history can be found in Table 2 and 3). Females are shown in red and males in blue.

\section{PRO hypertrophic}

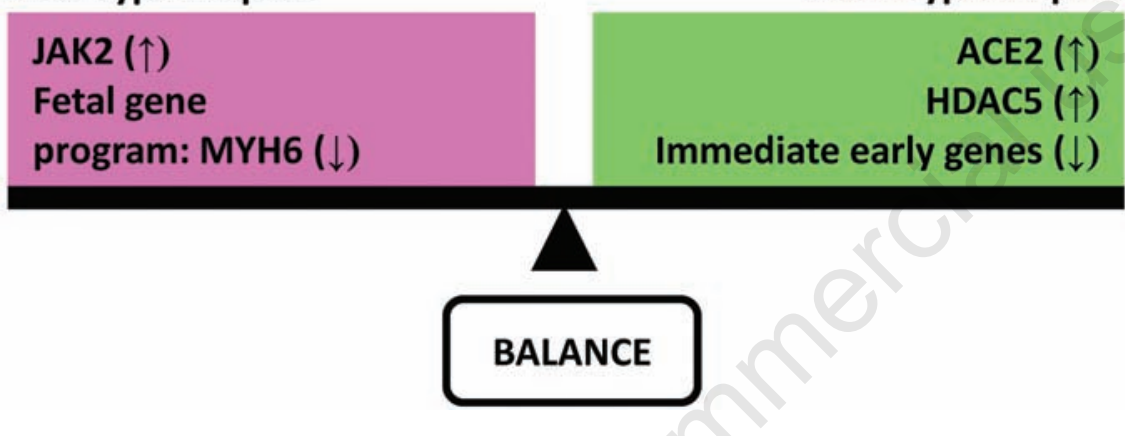

Figure 3. The balance of pro (pink box) and anti (green box) hypertrophic genes, pathways and mechanisms. Pro hypertrophic mechanisms are highlighted by the transcriptional down-regulation of $M Y H 6$, indicating that the switch to the fetal gene program is maintained, and the transcriptional up-regulation of JAK2 in the JAK-STAT pathway. The anti hypertrophic mechanisms were seen as a transcriptional down-regulation of the IEGs, FOS and EGR1, and a transcriptional up-regulation of $A C E 2$ and $H D A C 5$. $\uparrow$ and $\downarrow$ indicates change in gene expression in patients compared to controls

ment of a compensatory hypertrophy needed to adjust to the acute dysfunction of the heart. It seems that the observed regulation of the corresponding genes, with up-regulation of $A C E 2$ and no differential regulation of $A C E$, reflects an anti-hypertrophic response in the chronic state hypertrophy that is maintaining a balance between pro- and anti-hypertrophic effects/forces. ${ }^{29-30}$ The observed anti-hypertrophic ACE/ACE2 balance does not support the use of $A C E$-inhibitors as left ventricular (LV) remodelling treatment in HCM. This is in line with current therapy guidelines for HCM, where $A C E$-inhibitors are only recommended in a minority of the patients for treatment of end-stage heart failure ${ }^{31}$
Staging of the hypertrophy as a selection tool for treatment may be of clinical importance, as indicated by a recent experimental study by Seidman et al. They reported that losartan seemed to be of value in delaying the hypertrophic process in the early stages, but was without effect in reversing an already manifest cardiac hypertrophy. ${ }^{32}$ In children with HCM, progression of LVH can be attenuated by high doses of beta blockers which has so far not been shown in adults. Such studies may result in future combination therapies to induce regression of the hypertrophy. Although myocardial biopsy is not a routine procedure, such gene expression data could be of value to determine the stage of the disease.
In summary, this is a whole genome expression study comparing myectomies from nonend stage HOCM patients and controls. The data show evidence for both pro- and antihypertrophic transcriptional regulations, which suggests the possibility of adjusting the pharmacological treatment according to the stage of hypertrophy. An interesting aspect is the simultaneous up-regulation of the ACE2 gene and the ANP and BNP genes (NPPA and $N P P B) . A N P$ and BNP have diuretic and anti hypertrophic effects while $A C E 2$ has a repressive effect on RAAS ${ }^{33-34}$ This can be interpreted as an auto medication of the hypertrophic heart in the chronic disease state, contributing to an endogenous protection system, neither growing nor suppressing the already hypertrophic myocardium (Figure 3).

\section{Limitations}

Differences between the two methods, Illumina microarray and real time PCR, can be explained by the fact that the handling of the RNA before hybridization is more extensive compared to the single RT-PCR step before the real time PCR. This could possibly cause more sample variation in the microarray analysis. Another limiting factor for this study was that the size of the biopsies/myectomies only allowed for the Illumina micro array and four real time PCR runs.

\section{References}

1. Richardson P, McKenna W, Bristow M, et al. Report of the 1995 World Health Organization/International Society and Federation of Cardiology Task Force on the Definition and Classification of cardiomyopathies. Circulation 1996;93:841-2.

2. Maron BJ, Nichols PFr, Pickle LW, et alPatterns of inheritance in hypertrophic cardiomyopathy: assessment by M- mode and two-dimensional echocardiography. Am J Cardiol 1984;53:1087-94.

3. St John Sutton MG, Lie JT, Anderson KR, et al. Histopathological specificity of hypertrophic obstructive cardiomyopathy. Myocardial fibre disarray and myocardial fibrosis. Br Heart J 1980;44:433-43.

4. Varnava AM, Elliott PM, Sharma S, et al. Hypertrophic cardiomyopathy: the interrelation of disarray, fibrosis, and small vessel disease. Heart 2000;84:476-82.

5. Wisten A, Forsberg H, Krantz P, Messner T. Sudden cardiac death in 15-35-year olds in Sweden during 1992-99. J Intern Med 2002;252:529-36.

6. Booz GW, Day JNE, Baker KM. Interplay 
between the cardiac renin angiotensin system and JAK-STAT signaling: Role in cardiac hypertrophy, ischemia/reperfusion dysfunction, and heart failure. J Mol Cell Cardiol 2002;34:1443-53.

7. Rajabi M, Kassiotis C, Razeghi P, Taegtmeyer H. Return to the fetal gene program protects the stressed heart: a strong hypothesis. Heart Fail Rev 2007;12: 331-43.

8. Glennon PE, Sugden PH, Poole-Wilson PA. Cellular mechanisms of cardiac hypertrophy. Br Heart J 1995;73:496-9.

9. Lim DS, Roberts R, Marian AJ. Expression profiling of cardiac genes in human hypertrophic cardiomyopathy: insight into the pathogenesis of phenotypes. J Am Coll Cardiol 2001;38:1175-80.

10. Hwang JJ, Allen PD, Tseng GC, et al. Microarray gene expression profiles in dilated and hypertrophic cardiomyopathic end-stage heart failure. Physiol Genomics 2002;10:31-44.

11. Rajan S, Williams SS, Jagatheesan G, et al. Microarray analysis of gene expression during early stages of mild and severe cardiac hypertrophy. Physiol Genomics 2006; 27:309-17.

12. Maron BJ, Spirito P, Wesley Y, Arce J. Development and progression of left ventricular hypertrophy in children with hypertrophic cardiomyopathy. N Engl J Med 1986;315:610-4.

13. McKenna WJ, Spirito P, Desnos M, et al. Experience from clinical genetics in hypertrophic cardiomyopathy: proposal for new diagnostic criteria in adult members of affected families. Heart 1997;77:130-2.

14. Romhilt DW, Estes EH Jr. A point-score system for the ECG diagnosis of left ventricular hypertrophy. Am Heart J 1968;75: 752-8.

15. Perez S, Royo LJ, Astudillo A, et al. Identifying the most suitable endogenous control for determining gene expression in hearts from organ donors. BMC Mol Biol 2007;8:114.

16. Jackson JE. A user's guide to principal components. New York: John Wiley \& Sons, Inc.; 1991.

17. Wold S, Esbensen K, Geladi P. Principal components analysis Chemometr Intell Lab Syst 1987;2:37-52.

18. Yamazaki T, Komuro I, Kudoh S, et al. Endothelin-1 is involved in mechanical stress-induced cardiomyocyte hypertrophy. J Biol Chem 1996;271:3221-8.

19. Lijnen P, Petrov V. Renin-angiotensin system, hypertrophy and gene expression in cardiac myocytes. J Mol Cell Cardiol 1999;31:949-70.

20. Backs J, Olson EN. Control of cardiac growth by histone acetylation/deacetylation. Circ Res 2006;98:15-24.

21. Barry SP, Davidson SM, Townsend PA. Molecular regulation of cardiac hypertrophy. Int J Biochem Cell Biol 2008:40:202339.

22. Razeghi P, Young ME, Alcorn JL, et al. Metabolic gene expression in fetal and failing human heart. Circulation 2001;104: 2923-31.

23. Taegtmeyer H, Sen S, Vela D. Return to the fetal gene program: a suggested metabolic link to gene expression in the heart. Ann N Y Acad Sci 2010;1188:191-8.

24. Gupta MP, Gupta M, Zak R, Sukhatme VP. Egr-1, a serum-inducible zinc finger protein, regulates transcription of the rat cardiac alpha-myosin heavy chain gene. J Biol Chem 1991;266:12813-6.

25. Schiaffino S, Samuel JL, Sassoon D, et al. Nonsynchronous accumulation of alphaskeletal actin and beta-myosin heavy chain mRNAs during early stages of pressure-overload--induced cardiac hypertrophy demonstrated by in situ hybridization. Circ Res 1989;64:937-48.

26. Uozumi H, Hiroi Y, Zou Y, et al. gp130 plays a critical role in pressure overload-induced cardiac hypertrophy. J Biol Chem 2001; 276:23115-9.

27. Marrero MB, Schieffer B, Paxton WG, et al. Direct stimulation of Jak/STAT pathway by the angiotensin II AT1 receptor. Nature 1995;375:247-50.

28. Keidar S, Kaplan M, Gamliel-Lazarovich A. ACE2 of the heart: From angiotensin I to angiotensin (1-7). Cardiovasc Res 2007; 73:463-9.

29. Zisman LS. ACE and ACE2: a tale of two enzymes. Eur Heart J 2005;26:322-4.

30. Donoghue M, Hsieh F, Baronas E, et al. A novel angiotensin-converting enzymerelated carboxypeptidase (ACE2) converts angiotensin I to angiotensin 1-9. Circ Res 2000;87:E1-9.

31. Maron BJ, McKenna WJ, Danielson GK, et al. American College of Cardiology/ European Society of Cardiology Clinical Expert Consensus Document on Hypertrophic Cardiomyopathy. A report of the American College of Cardiology Foundation Task Force on Clinical Expert Consensus Documents and the European Society of Cardiology Committee for Practice Guidelines. Eur Heart J 2003;24: 1965-91.

32. Teekakirikul P, Eminaga S, Toka 0, et al. Cardiac fibrosis in mice with hypertrophic cardiomyopathy is mediated by nonmyocyte proliferation and requires Tgfbeta. J Clin Invest 2010;120:3520-9.

33. Kinoshita H, Kuwahara K, Nishida M, et al. Inhibition of TRPC6 channel activity contributes to the antihypertrophic effects of natriuretic peptides-guanylyl cyclase-A signaling in the heart. Circ Res 2010;106: 1849-60.

34. Rosenkranz AC, Woods RL, Dusting GJ, Ritchie RH. Antihypertrophic actions of the natriuretic peptides in adult rat cardiomyocytes: importance of cyclic GMP. Cardiovasc Res 2003;57:515-22. 